\title{
Educación pública, autonomía universitaria y cambio político: notas para el análisis del movimiento universitario en chile, $2011^{1}$
}

\author{
José Molina Bravo² \\ Universidad de Arte y Ciencias Sociales (Chile) \\ Universidad Autónoma de Colombia (Colombia) \\ Grupo de investigación HISULA \\ jamolinab@gmail.com
}

Recepción: 04/03/2013

Evaluación: 15/05/2013

Aceptación: 28/06/2013

Artículo de Reflexión

DOI: http:/ / dx.doi.org / 10.9757 / Rhela. 21.08

\section{RESUMEN}

El trabajo propone tópicos de análisis e interpretación de las movilizaciones estudiantiles desarrolladas en Chile durante el año 2011, las que reposicionaron en el centro del debate político nacional, el valor y necesidad de fortalecer la Educación Pública, cuestionando las políticas y reformas neoliberales operadas desde 1981 y profundizadas durante los gobiernos democráticos del período 1990-2010. Analiza desde la perspectiva de la historia de la educación, el rol protagónico de la Universidad, tanto en la capacidad organizativa y política, como en las reivindicaciones propuestas por el movimiento social universitario.

Palabras clave: Revista Historia de la Educación Latinoamericana Educación pública, autonomía universitaria, Movimiento social universitario, conflicto universitario.

1 Este trabajo corresponde a una ampliación de la ponencia presentada en el Panel "Reformas y Movimientos Universitarios" del Seminario Internacional VENDIMIA, RUDECOLOMBIA, Tunja 7 de noviembre de 2012. Esta ponencia se desarrolló en el marco de la Pasantía de Investigación en el Doctorado en Ciencias de la Educación, Universidad Pedagógica y Tecnológica de Colombia y se articula al proyecto, Reformas y movimientos universitarios en Colombia SGI 1295 desarrollado por el grupo Ilustración en América Colonial - ILAC y financiado por la Dirección de Investigaciones de la Universidad Pedagógica y Tecnológica de Colombia.

2 Doctorando del programa Doctorado en Procesos Sociales y Políticos de América Latina de la Universidad de Arte y Ciencias Sociales, Magíster en Sociología, Docente e Investigador, de la Facultad de Ciencias Humanas, Programa Historia, Universidad Autónoma de Colombia e integrante del grupo de investigación HISULA de la Universidad Pedagógica y Tecnológica de Colombia. 
Public Education, university autonomy and political change: Notes for the analysis of the university movement in Chile, 2011"

\section{ABSTRACT}

The paper proposes topics of an analysis and interpretation of student mobilizations carried out in Chile during 2011, which replaced in the center of the national political debate, the value and the need to strengthen public education, challenging policies and neoliberal reforms developed since 1981 and deepened during the democratic governments of the period 1990-2010. It analyzes from the perspective of the history of education, the main role of the university in both the organizational and political capacity, as in the claims proposed by the university social movement.

Key words: Journal of the History of American Education, public education, university autonomy, academic social movement, university conflict.
Educação pública, autonomia universitária e mudança política: Notas para a análise do movimento universitário no Chile, 2011

\section{RESUMO}

O trabalho propõe tópicos de analise e interpretação das mobilizações estudantis desenvolvidas no Chile durante o ano de 2011, as que recolocaram no centro do debate nacional o valor e a necessidade de fortalecer a Educação Pública, questionando as políticas e reformas neoliberais operadas desde 1981 e aprofundadas durante os governos democráticos do período 1990-2010. Analisa a partir da perspectiva da história da educação, o papel protagonista da Universidade tanto na capacidade organizativa e política como nas reinvindicações propostas pelo movimento social universitário.

Palavras-chave: Revista História da Educação Latino-americana, Educação pública, autonomia universitária, Movimento social universitário, conflito universitário.

\section{INTRODUCCIÓN}

Las movilizaciones estudiantiles y sociales que se desarrollaron en Chile el año 2011 se convirtieron en un analizador histórico de la sociedad chilena de las últimas cuatro décadas. Tales acciones colectivas expresaron motivos, intereses y propuestas de cambio del sistema educativo nacional, traspasaron el límite de 'lo universitario' y de 'lo escolar' e impactaron tanto la matriz sociopolítica democrática-neoliberal como la composición cultural y generacional de los discursos críticos y de cambio sobre el orden y funcionamiento social. La Universidad se reposicionó como un sujeto que, consciente de su crisis, interpeló al conjunto de la sociedad.

Se propone un análisis a partir de tres ejes de reflexión: en primer lugar, la noción de Educación Pública como un tópico de convergencia de los discursos en el movimiento social-universitario que refleja, primordialmente, la cohesión interna-estamental de la idea de Universidad encarnada en la 
Universidad de Chile; segundo, el ámbito del cambio político ${ }^{3}$ inscrito en la lógica argumental y de reorientación institucional propuesta por las organizaciones estudiantiles agrupadas en la Confederación de Estudiantes de Chile (CONFECH) y, también, de los Rectores de Universidades Estatales y de Rectores de Universidades Tradicionales; y, en tercer lugar, se revisará el reposicionamiento de la noción y/o principio de autonomía universitaria como ámbito de distinción política y diferenciación funcional de la Universidad Estatal-Pública, en el contexto actual del Sistema de Educación Superior en Chile. ${ }^{4}$

\section{Conflicto universitario: contexto institucional y criterios heurísticos}

Se presentan las características del conflicto social universitario chileno, del año 2011, considerando, por una parte, el contexto histórico-institucional que aborda en términos generales la trayectoria del sistema universitario chileno y, por otra, la interpretación del mismo conflicto desde la perspectiva de la historia de la educación.

\section{Universidad y Estado-Docente en Chile: descripción general del con- texto institucional}

El reconocimiento de la Educación como un mecanismo de intervención en la sociedad, está presente en el Estado republicano chileno desde la Constitución de 1833, que señaló la preocupación por la formación de ciudadanos disciplinados e instruidos (en un contexto autoritario). Luego, con la Ley del 1 de Febrero 1837, la institucionalidad educativa estatal tomó forma con el Ministerio de Justicia, Culto e Instrucción Pública. ${ }^{5}$ No obstante,

3 La noción de cambio político está asociada a la articulación entre conflicto y cambio, planteada por Charles Tilly. Sobre los efectos de influencia entre conflicto político y cambio social, Tilly sistematiza cuatro categorías: "1. Reorganización: El esfuerzo del conflicto transforma las relaciones sociales internas y externas de los actores implicados, incluyendo autoridades, terceras partes y el objeto de sus reivindicaciones. 2. Realineamiento: Más concretamente, la lucha, la defensa y la cooptación alteran las alianzas, rivalidades y enemistades entre gobernantes, otros contendientes y los grupos revindicativos. 3. Represión: Los esfuerzos de las autoridades en la represión o consentimiento de los que los desafían producen cambios directos -la declaración de poderes de emergencia- e indirectos - efectos en los gastos de vigilancia, actividad policial y fuerzas militares- en el ejercicio del poder. 4. Realización: Los demandantes exigen cambios específicos, negocian con éxito con los detentadores del poder y hasta los desplazan". Charles Tilly. "Conflicto político y cambio social". En Los movimientos sociales. Transformaciones politicas y cambio cultural, editado por Pedro Ibarra y Benjamín Tejerina (Madrid: Editorial Trotta, 1998), 37-38.

4 En términos metodológicos, estas notas han sido construidas mediante el análisis crítico del discurso, es decir, de revisión de las prácticas textuales, discursivas y sociales observables y atribuibles al conflicto sociopolítico que hoy representa la Reforma en Educación que se propone desde la sociedad civil chilena y, en particular, desde asociaciones y agrupaciones que posicionan a la Universidad y a la Educación como un medio -un espacio institucional- de cambio social. Al respecto, Norman Fairclough. El análisis crítico del discurso y la mercantilización del discurso público: las universidades. (Buenos Aires: Discurso y Sociedad, 2008). Consulta 30 de octubre de 2012 http://www.dissoc.org/ediciones/v02n01/DS2\%281\%29Fairclough.pdf

5 Las funciones del Ministerio, en el ámbito de la Instrucción Pública, fueron: "Dirigir la educación en todo el territorio nacional; 
es con la fundación de la Universidad de Chile en 1842 que el Estado Docente se articula en niveles y funciones de regulación.

La Universidad de Chile dio forma al propósito del Estado de racionalizar y reformar la sociedad chilena desde la generación y transmisión del conocimiento; ${ }^{6}$ tuvo como referencia el modelo de universidad napoleónica, convirtiendo a la Universidad en Superintendencia, con lo cual se configuró una institucionalidad acorde con las necesidades del Estado. ${ }^{7}$ Durante gran parte del Siglo XIX, principalmente durante la República Conservadora (1833-1860), la función otorgada a la Universidad de Chile gozó del consenso político sobre las atribuciones del Estado en el ámbito educativo; liberales y conservadores mantuvieron acuerdo en los principios que orientaron tanto la fundación de la Universidad como el valor del conocimiento en la construcción de la Nación.

La Universidad se definió, fundamentalmente, como docente, pese a las tensiones sufridas con relación al valor universitario de la investigación y también las perspectivas sobre las formas adecuadas de organización del trabajo académico; se mantuvo la orientación hacia la formación de profesionales y la enseñanza de conocimientos útiles y prácticos. ${ }^{8}$

En la perspectiva de J. J. Brunner, el sistema de educación superior entre 18421967 tuvo un carácter elitista, no tanto por los valores, sentidos y perfiles de la población que accedía a ella, como por el lento crecimiento de la matrícu-

Inspeccionar establecimientos; Promover el desarrollo de la educación; preocuparse de la economía, policía y fomento de los establecimientos de Estado y municipales; Otorgar autorización para abrir escuelas; Becar alumnos; Enviar socorros, recompensas o pensiones a profesores del sector público o particular; y Promover expediciones científicas". Jorge Insunza, $L a$ construcción del derecho a la Educación y la institucionalidad educativa en Chile (Santiago: OPECH, 2009), 24.

6 Sol Serrano, Universidad y Nación (Santiago: Editorial Universitaria, 1994)

7 Las siguientes medidas estatales para la administración educacional fueron la creación del Consejo de Instrucción Pública en 1844 y, fundamentalmente, la promulgación de la Ley General de Instrucción Primaria, en 1860. Serrano, Universidad y Nación, 78

8 En el siglo XX, esta orientación profesional de la Universidad fue criticada por la Federación de Estudiantes de la Universidad de Chile (FECH), al manifestar que el vínculo de esta con los propósitos estatales de desarrollo nacional desvirtuaba el carácter de la Universidad y su verdadero sentido: responder a las finalidades individuales y colectivas. El Manifiesto Pro-Reforma Universitaria de 1922 señaló: "Ha sido hasta aquí el Estado el encargado de la organización general de la Universidad y quien, de una manera indirecta pero certera, le ha impuesto sus normas directas. Nosotros sostenemos y sostendremos con la fuerza tenaz de los que tienen la razón, que la organización de la Universidad debe resultar de la voluntad de los que la constituyen actualmente, alumnos y profesores, y de aquellos que habiendo pasado por sus aulas mantienen con ella vínculos constantemente renovados. Queremos, por lo tanto, estar representados en los consejos para hacemos oír y para establecer relaciones verdaderas de simpatía y mutua comprensión entre los que enseñan y los que aprenden. La representación del alumnado en los organismos representativos es una de nuestras aspiraciones inmediatas." Emir Sader, Pablo Gentili y Hugo Aboites, La Reforma Universitaria. Desafios y perspectivas 90 años después, (Buenos Aires: CLACSO, 2008), 200-201. Es importante señalar que esta crítica contra el carácter profesional de la Universidad de Chile se desarrolló en el convulso ambiente sociopolítico de la década de 1920; en el marco de una creciente institucionalización del desarrollo y conflictos 'internos' del Estado Docente chileno, bajo la dictadura de Carlos Ibáñez del Campo. 
la en el núcleo institucional tradicional de universidades, ${ }^{9}$ conformando un sistema universitario simple. ${ }^{10}$ Entre 1967-1973, este sistema, en coherencia con las tendencias internacionales, experimentó una expansión de la matrícula, el aumento de la diferenciación interna por actividades de extensión e investigación y, muy marcadamente, la politización de la Universidad en el contexto de la Reforma Universitaria. Este proceso de consolidación del sistema universitario chileno reflejó la continuidad del patrón de relación entre el Estado y la Universidad, a la manera de un pacto histórico fundado en la autonomía universitaria, el financiamiento estatal a universidades tradicionales y el valor funcional de la educación.

Tal modelo de coordinación del sistema universitario, basado en el pacto histórico mencionado, fue transformado luego del golpe de Estado de 1973; en el contexto del experimento neoliberal, el funcionamiento y sentido de las universidades fueron integradas a las modernizaciones sectoriales que, en conjunto, buscaron instituir una sociedad basada en libertades económicas en un régimen de libertades restringidas. Así, la modernización de la educación fue impactada por la transformación del Estado subsidiario de la demanda por educación. ${ }^{11}$

A la fase de depuración ideológica de las Universidades y la anulación de las modificaciones producidas por la Reforma de 1967, siguieron las iniciativas de cambio y control de funciones y a la definición de la responsabilidad universitaria por desempeños y usos adecuados de recursos públicos, lo que se convirtió en el contexto de introducción de las nociones de calidad. En el período 1980-1981 se dictó un conjunto de decretos que consolidó el cambio en el patrón de relaciones entre Estado y Universidad: se impuso la descentralización y la lógica de financiamiento de mercado (cobro de aranceles, aportes fiscales directo, aporte fiscal indirecto, venta de servicios); la segmentación del sistema de educación superior entre instituciones

9 Este núcleo de universidades tradicionales fue conformado por dos universidades estatales: Universidad de Chile (1845) y Universidad Técnica del Estado (1947) 6 privadas Universidad de Concepción (1919), Universidad Austral (1954), Universidad Técnica Federico Santa María (1931), Pontificia Universidad Católica de Chile (1888), Universidad Católica de Valparaíso (1928) y Universidad del Norte (1956).

10 Así Brunner: "Las universidades formadas durante este tiempo aspiran a obtener y obtienen cierto grado de igualdad, independientemente, de su estatuto jurídico que las distingue como universidades estatales, universidades privadas confesionales (católicas) y universidades privadas laicas. Todas terminan percibiendo recursos del Estado, recursos que forman casi la totalidad de su presupuesto, y reciben -junto con el reconocimiento oficial de su autonomía- el derecho a expedir títulos profesionales y grados académicos". José Joaquín Brunner, Educación Superior en Chile. Instituciones, mercados y politicas gubernamentales (1967-2007) (Santiago: Ediciones Universidad Diego Portales, 2009), 170

11 Carlos Ruiz, De la República al Mercado (Santiago: LOM, 2010). 
tradicionales y privadas y, finalmente, se impuso la Libertad de Enseñanza por sobre el derecho a la Educación.

\section{La 'crisis' de la universidad: ¿institución educativa o mecanismo de politica social?}

La integración subordinada de los sistemas nacionales de Educación a las directrices de organismos financieros económicos multilaterales, principalmente, así como la regulación desde agrupaciones y corporaciones de rango subnacional, han transformado las capacidades estatales y de los sistemas universitarios para regular la existencia, funcionamiento, clasificación, evaluación de instituciones de educación superior, lo que impacta negativamente el rol del Estado en la garantización del derecho a la educación y al conocimiento. Además de complejizar los contenidos y procedimientos sociopolíticos y propiamente académicos y científicos, para la definición de la función de la Universidad en la sociedad y sus proyectos de desarrollo, regulación y/ o emancipación. ${ }^{12}$

Con la transformación neoliberal de la Universidad y del Estado, se produjeron dos cambios categoriales centrales: primero, la comprensión del vínculo entre estas dos instituciones y los individuos desde la lógica del mercado universitario, considerando al privatismo y la mercadización como descriptores del funcionamiento del sistema universitario; la legitimidad categorial (y sociopolítica) de este desplazamiento se basó en la consideración de que todas las Universidades son públicas dado el reconocimiento que históricamente recibieron del Estado, en el marco de su patrón histórico de relación, por ende, la crítica al Estado subsidiario sería más bien una reacción defensiva de la Universidad pública-estatal. ${ }^{13}$

12 Al respecto, Luis Riveros, exrector de la Universidad Chile, planteó: "En este caso también prevalece una crisis de calidad y de medios, que se refleja en una alta varianza tanto en aspectos formativos como en la profundidad y relevancia de la investigación. La política del Estado no puede dejar de regular en forma más efectiva sobre estos temas, especialmente cuando existen recursos de importancia que se dedican a las universidades. No es posible que la política conduzca a una absoluta falta de regulación sobre estándares de gestión y resultados en el caso de las universidades con subsidio fiscal directo, mientras que en el sector privado no tradicional prevalece una confusa mezcla de calidades. En particular, no es posible que el Estado deje en abandono a sus propias universidades, especialmente por medio de políticas que tienden a dejar en mayor desmedro a las más complejas, las que poseen un mayor capital humano y físico desarrollado, precisamente, sobre la base del esfuerzo histórico de la política pública por contar con una investigación y una formación profesional y de posgrado de primera línea. Hay que recuperar el sentido nacional de largo plazo en el diseño de la política universitaria y educacional del Estado". Luis Riveros, La Universidad de Chile hacia el 2010. Estrategia y Acción para un Desarrollo Sostenible (Santiago: Universidad de Chile, 2010). Consulta 10 de octubre de $2012 \mathrm{http} / /$ www.uchile.cl/portal/presentacion/historia/luis-riveros-cornejo/publicaciones/7753/iv33-estado-docente

13 Brunner y Uribe plantean que la noción de privatismo se basa en dos variables: el porcentaje de alumnos que se matriculan en instituciones privadas de educación superior (privatismo de la matrícula) y el porcentaje del gasto total en educación superior que proviene de fuentes privadas (privatismo de los recursos); mientras que la noción de mercadización refiere a la dinámica de los sistemas de educación superior que, dado su nivel de privatismo, coordinan sus actividades mediante el mercado, competencia 
El segundo cambio categorial relevante consiste en la adecuación del funcionamiento de la Universidad y del denominado "mercado universitario", conforme el principio de equidad, lo que transforma a la institucionalidad universitaria y sus procesos formativos en componentes de un tipo de política social relacional. El carácter social de esta política corresponde al reconocimiento del derecho de cada persona a educación de calidad (información de decisión de inversión, focalización de becas y créditos, vulnerabilidad social, pacto social); a la función garantista del Estado sobre los resultados educativos (perfiles de ingreso, cambios curriculares, nivelación de competencias, acompañamiento estudiantil); la inserción en el mundo laboral (empleabilidad, señalización, segmentación por prestigios académicos de los certificados y capital reputacional de las universidades); y, con todo, al cumplimiento de la igualdad de condiciones y derechos de los individuos. ${ }^{14}$

Por su parte, el carácter relacional ${ }^{15}$ de la política desplaza la problematización de la crisis de la universidad desde la legitimación de sus saberes y de su autonomía hacia su función en el campo de producción de conocimientos científicos y profesionalización, en el cual el vínculo entre el Estado y los individuos, mediado por la Universidad, se especifica en la propia mediación de determinadas comunidades académicas. En el marco de un Estado Docente regulador del mercado universitario y no-interventor del campo organizacional estructurado sobre la 'libertad de enseñanza', la autonomía universitaria se concibe aun como un principio de intervención sobre individuos y de generación de experiencias de individualización y de prácticas culturales y sociales. Sin embargo, esto debe ser contextualizado por la función mediadora hegemónica de la Universidad en la sociedad del conocimiento y, a la vez, en la valorización del conocimiento en el marco del capitalismo cognitivo; tal función consiste en incluir a los individuos que acceden a la Universidad en una economía-política, esto es, en una lógica cognitiva y productiva crecientemente mercantilizada. ${ }^{16}$

por alumnos, recursos y prestigio con una intervención a distancia por parte del Estado y las políticas gubernamentales. José Joaquín Brunner y Daniel Uribe, Mercados universitarios: el nuevo escenario de la educación superior (Santiago: Ediciones Universidad Diego Portales, 2007), 183 y 188.

14 Oscar Espinoza y Luis González, "Políticas de Educación Superior en Chile desde la perspectiva de la Equidad", Sociedad y Economía 22 (2012): 69 - 94.

15 Desde la perspectiva relacional, la "inclusión indica en cualquier caso una pertenencia, un reconocimiento, un criterio de selección. Por ello, inclusión significa la posibilidad de acceder a un sistema societario cuando se reúnen determinadas características. El hecho de indicar que se posee una determinada propiedad o cualidad significa trazar una distinción respecto de quien no la posee (y que, por lo tanto, queda excluido)" Pierpaolo Donati, "Nuevas políticas sociales y Estado social relacional" Revista Española de Investigaciones Sociológicas 108 (2004): 11.

16 J. F. Lyotard, La condición posmoderna. Informe sobre el saber (Madrid: Editorial Cátedra, 1987). 


\section{El conflicto social-universitario desde el enfoque de historia de la educación: criterios heurísticos}

En la perspectiva de la historia de la educación, ${ }^{17}$ la descripción y análisis historiográfico de los conflictos y reformas universitarias en América Latina debe considerar cinco criterios, que se plantean a continuación:

a. Los fines asociativos que se expresan en la conflictividad educativa y universitaria impactan en campos más amplios de relaciones sociales, productivas, sociopolíticas y, fundamentalmente, culturales. En este sentido, el conflicto y movimiento social-universitario chileno amplificó el impacto de las situaciones de endeudamiento de las familias y de los estudiantes, que a la vez representan características propias del privatismo, al instalar un cuestionamiento a la lógica financiera-bancaria de la subsidiariedad, reafirmando la percepción y denuncia del lucro en educación y la concepción de la educación como bien de consumo, imperante en el diseño de funcionamiento del sistema universitario chileno.

b. La "acción educativa" se enmarca en una política pública propia de los Estados Nacionales; así, la crítica de los fines educacionales y del funcionamiento de la institucionalidad educacional se relaciona directamente con la operación de los gobiernos, los partidos políticos y la sociedad civil, con lo cual se erige una escena y espacio público delineada por los conflictos y sus expresividades simbólicas y materiales. En este marco, la politización del movimiento universitario chileno alcanzó una dimensión social más amplia al establecer en el centro de su discurso la definición de la educación como un bien público, complementando su irrupción en la escena de la política educacional y social, con el posicionamiento de la función de la universidad en el desarrollo nacional, expresado en el ámbito de la ciencia y el desarrollo material, en la formación de ciudadanos, y de intelectuales, profesionales y técnicos. ${ }^{18}$

17 En este apartado se siguen los planteamientos de Álvaro Acevedo. Al respecto, ver Alvaro Acevedo, "Una heurística para el estudio de la historia de las reformas y conflictos en la universidad latinoamericana". Revista Historia de la Educación Colombiana 8 (2005).

18 "Las Universidades Estatales, por la naturaleza de su misión (ser conciencia crítica) y la nobleza de su tarea (producir y transmitir el conocimiento preparando nuevas generaciones para el desarrollo del país), son instituciones de carácter estratégico para el desarrollo de ciertos valores universales, que le dan sentido trascendente y colectivo a tal desarrollo, tales como la libertad, la igualdad y la democracia; los derechos humanos, los derechos ciudadanos, la tolerancia y la no discriminación; la confianza, la solidaridad y la ética pública". Consorcio de Univesidades del Estado de Chile. Nuevo trato con el Estado. Fortaleciendo la política de Educación Superior Estatal (Santiago: CUECH, 2009), 12. 
c. Los conflictos educativos-universitarios y los cambios que estos promovieron se desplegaron en el plano de la cultura, reflejando la artificialidad de distinciones entre tipos de conocimientos $y$, sobre todo, la potencia de los imaginarios sociopolíticos vinculados al movimiento estudiantil chileno. Así, el movimiento social-universitario expresó nuevas formas de politización en las que se destacan la posición y dinámica generacional y, también, la modificación de repertorios de protesta y expresividad crítica de las movilizaciones. Existen, entonces, ejemplos de la pervivencia de una memoria histórica expresiva de conflictividad que ha implementado un espacio de expresión, circulación y creación de críticas a la reformas neoliberales operadas en Chile desde 1973.

d. Las escalas de los conflictos sociales-educativos implican a la región, la nación y también la "red de los influjos externos". Este argumento inserta el conflicto social universitario chileno dentro de las experiencias de movimientos sociales latinoamericanos críticos del neoliberalismo. ${ }^{19}$ Así mismo, este conflicto se inserta en la constelación de acciones y transformaciones sociopolíticas generadas por la denominada Revolución de los Pinguinos y la propuesta de desmunicipalización de la educación. ${ }^{20}$

A lo anterior se suma el refuerzo de la compresión de la educación como un derecho social, lo cual posibilitó y proyectó la problematización del principio de subsidiariedad estatal como un abandono por parte del Estado de su rol de garante en el ejercicio de derechos y de incumplimiento de los tratados internacionales que Chile ha suscrito, específicamente, el Pacto Internacional de Derechos Económicos, Sociales y Culturales (ratificado por Chile en 1969, aborda la obligación del Estado en el financiamiento de la Educación y educación gratuita progresiva para enseñanza secundaria y universitaria) y el Pacto de San José de Costa Rica (que permite demostrar la subordinación del Derecho a la Educación a la Libertad de Enseñanza). ${ }^{21}$

19 José Seoane, Clara Algranati y Emilio Taddei, "Realidades y desafíos políticos de "Nuestra América" Revista América Latina 11 (2011).

20 No obstante, en este punto es necesario recalcar la importancia del ingreso a la OCDE de Chile, como miembro número 31; esto ofreció un patrón de comparación utilizado para comparar el gasto público en educación y concentrar los esfuerzos de influenciar la composición y alianzas políticas, para el rechazo o aprobación del presupuesto público 2012 y 2013. Al respecto se planteó: "se concluye que la inversión pública en Chile para enfrentar los desafíos en Educación Superior dista aún mucho del esfuerzo que realizan los países de la OECD y expone en cifras muy elocuentes el acentuado carácter privatizador de la política chilena". Consorcio de Universidades el Estado de Chile, Nuevo Trato con el Estado, 12.

21 Al respecto la Confech apuntó: "La conclusión final nos lleva a señalar enfáticamente que más allá de todo formalismo jurídico interno, el Estado chileno está obligado a responder a la comunidad internacional sobre la problemática en torno a la crisis del modelo educativo actualmente vigente. La perspectiva internacional es un imperativo jurídico del cual se debe hacer cargo." 
e. La conflictividad educativa-universitaria requiere de teorías que expliquen el tipo de acción colectiva que se hace visible en la esfera educativa. En este sentido, el movimiento social-universitario y, sobre todo, su articulación con actores sociales territoriales, generacionales, políticos, entre otros, activaron el debate sobre la acción histórica y el cambio social en Chile. ${ }^{22}$

No obstante, uno de los discursos que 'innovaron' en la composición de las lógicas expresivas y discursivas del movimiento social-universitario fue la comprensión y valoración de las ideas de calidad y excelencia académica como parámetros de la garantización del ejercicio de derechos. Este aspecto dio consistencia a la interpelación que hacia el Estado dirigió la población y su asociación voluntaria y crítica; el Estado se vio abordado en su (posible) ideología garantista, en su (efectiva) disposición subsidiria y en la eficacia de sus enfoques y procedimientos sobre la calidad de la educación universitaria.

\section{Asociación hipótesis-enunciados observacionales: caso chileno}

Para efecto de esta descripción, consideraremos que las políticas públicas educativas nacionales se han orientado principalmente por una racionalidad instrumental que prioriza el binomio ciencia-desarrollo material como contenidos centrales del progreso social; esto ha implicado que la Educación (y la Universidad) se han desarrollado en la tensión simbólica, política e histórica de una modernización sin modernidad; asociando a los movimientos de estudiantes y educacionales críticos una agenda educativa, la cual en el caso chileno se compuso con los siguientes principios: ${ }^{23}$

Confederación de Estudiantes de Chile. Fundamentos para un sistema público gratuito de educación para Chile (Santiago: Confech, 2011), 14. (cursivas en el original)

22 Se destacan las propuestas e interpretaciones de la historiografía social-popular y su comprensión política de categorías como las de soberanía, historicidad, sujeto y proyecto histórico; estas circularon y orientaron los jucios críticos y los análisis de conjunto que, a la par de un movimiento social-universitario y un movimiento social de estudiantes secundarios, profundizaban la evidente obsolescencia de los partidos políticos y los liderazgos demócratas tradicionales.

23 Se siguen los planteamientos de Álvaro Acevedo respecto de la conflictividad universitaria. Acevedo, Una heurística para el estudio, 181-182. 


\section{Cuadro 1. Reinvindicaciones del movimiento social universitario}

\begin{tabular}{|c|c|}
\hline Principios & Caso chileno \\
\hline 1. Inclusión educativa & $\begin{array}{l}\text { Garantías de acceso, permanencia y resultados del } \\
\text { proceso de formación universitaria, orientadas } \\
\text { por el principio de equidad. }{ }^{1} \\
\text { La incorporación de la perspectiva multicultural } \\
\text { vinculada a la "Inclusión y respeto de los } \\
\text { derechos educativos y lingüísticos de los pueblos } \\
\text { indígenas". }{ }^{2} \\
\text { Crítica de la Prueba de Selección Universitaria } \\
\text { como mecanismo de selección, ya que reproduce } \\
\text { desigualdades y brechas educativas propias de la } \\
\text { Educación Primaria y Secundaria. }\end{array}$ \\
\hline $\begin{array}{l}\text { 2. Construcción de } \\
\text { sociedad civil }\end{array}$ & $\begin{array}{l}\text { La democratización interna de las Universidades } \\
\text { y Escuelas, las que en tanto organizaciones, deben } \\
\text { permitir y promover la participación de todos los } \\
\text { estamentos. }\end{array}$ \\
\hline $\begin{array}{l}\text { 3. Valoración de respeto } \\
\text { de lo público como base } \\
\text { de diálogo e interacción } \\
\text { con el Estado }\end{array}$ & $\begin{array}{l}\text { Esto determina, fundamentalmente, la misión de } \\
\text { la Universidad y la concepción de la educación } \\
\text { como un bien público. }\end{array}$ \\
\hline $\begin{array}{l}\text { 4. Desarrollo de } \\
\text { competencias para } \\
\text { la construcción de lo } \\
\text { público }\end{array}$ & $\begin{array}{l}\text { El propósito de los nuevos procesos formativos } \\
\text { del Sistema Nacional de Educación Pública } \\
\text { propuesto y su asociación al principio de } \\
\text { excelencia, como resultado de la asociación entre } \\
\text { calidad y pertinencia de las universidades. }\end{array}$ \\
\hline $\begin{array}{l}\text { 5. Reconocimiento de la } \\
\text { acción social colectiva y } \\
\text { del Estado de Derecho }\end{array}$ & $\begin{array}{l}\text { La realización de marchas, eventos masivos y } \\
\text { ocupación de diferentes vías de expresión, que } \\
\text { se confrontan a la criminalización de la protesta, } \\
\text { llevada adelante por el gobierno (como la } \\
\text { denominada Ley Hinzpeter). }\end{array}$ \\
\hline
\end{tabular}

Fuente: Elaboración del autor. 


\title{
3. Temporalidades del conflicto y desarrollo del movimiento social-universitario
}

En la dimensión coyuntural, el conflicto social-universitario reflejó fundamentalmente las lógicas de asociación y politización gremial y corporativo, referido al movimiento estudiantil-universitario y el desempeño de los Rectores de Universidades Estatales y Tradicionales.

En el caso del movimiento estudiantil, este conflicto se inscribió en la etapa iniciada el año 2006, que la propia Confech describe como el paso de "universitarios a actores políticos", lo cual se condensó en los siguientes objetivos:

\begin{abstract}
Construir un proyecto de educación garantizado constitucionalmente como un derecho social universal en todos sus niveles, fundado en un sistema de educación pública, democrática, pluralista, gratuita y de calidad, orientado a la producción de conocimiento para un desarrollo integral e igualitario y [...] Frenar la reforma privatizadora del gobierno en materia educativa y posicionar las demandas transversales: educación gratuita, fin al lucro, democratización, fin al endeudamiento y al autofinanciamiento y acceso equitativo. ${ }^{24}$
\end{abstract}

De igual forma, la temporalidad del accionar político de los Rectores de Universidades Estatales se basó en los contenidos del texto Nuevo Trato con el Estado elaborado por la Universidad de Chile, coincidente con la propuesta de "Pacto Social entre el Estado y sus Universidades", elaborada por el Consorcio de Rectores de Universidades Estatales de Chile, en el año 2009. Este documento plantea que

Chile carece de un modelo sustentable de universidad estatal pública en un mundo en que el conocimiento será la gran herramienta para asegurar un acceso a iguales oportunidades, a los frutos del progreso $y$ a una mejor calidad de vida de las chilenas y chilenos en el siglo XXI. ${ }^{25}$

Las temáticas del conflicto universitario-educacional, planteadas por los estudiantes y los Rectores de Universidades Estatales y Tradicionales, en el

24 Confederación de Estudiantes de Chile. "Historia de la Confech y el movimiento universitario chileno en los últimos 30 años". (Santiago: Confech, 2011). Consulta 5 de noviembre de 2012 http://www.filosofiauc.com/uploads/1/1/5/5/11555117/historia_de_ la confech y el movimiento universitario chileno en los ltimos 30 aos-1.pdf

25 Consorcio de Universidades del Estado de Chile, Nuevo trato con el Estado, 3. 
período 2011-2012, se sintetizaron en un hecho ocurrido el 26 de Julio de 2011, cuando se suscribió el documento "Puntos de Convergencia" entre el CRUCH y la Confech, ${ }^{26}$ que en el número 3 señala

"El CRUCH y la Confech comparten que i) la Educación Superior debe ser una Política de Estado, abordada con visión de largo plazo y definida con la participación de actores relevantes, avanzando sobre grandes acuerdos y con sentido de urgencia, ii) el sistema universitario debe ser reconocido por el Estado como un actor fundamental para el desarrollo social, cultural, educacional, humanístico, económico, científico y tecnológico del país, y iii) el Estado debe asumir el compromiso de aumentar significativamente el monto del financiamiento estatal al sistema de educación terciario de modo de llevarlo, en un periodo razonable, al menos al promedio de los países de la OECD y así no seguir gravando a las familias, especialmente a las más vulnerables y de la clase media".

Luego de la alianza temática y de puntos de vistas sobre la crisis y mejoramiento de la Educación Pública, el Consejo de Rectores de Universidades Chilenas enfatizó la valoración del movimiento estudiantil como protagonista del posicionamiento público del debate sobre educación, llamando también a deponer las movilizaciones que afectaban el funcionamiento de las Universidades en toma. Ofreció garantías para la continuidad de los debates internos y las movilizaciones, en armonía con el desarrollo de la actividad académica. En su evaluación de las movilizaciones, el Consejo de Rectores refrendó haber cumplido un rol de mediador entre el Gobierno y los estudiantes, tendiente al acercamiento de posiciones y la generación de consensos.

Durante el año 2012, el Consejo de Rectores de Universidades Chilenas estableció como temas "sustanciales en educación" la creación de la Superintendencia de Educación, el fin al lucro en educación y el aseguramiento de la calidad; estos temas fueron refrendados como temas comunes con los estudiantes, además de la demanda por democratización de las Universidades y la no criminalización del movimiento estudiantil. A esto se sumó la "convergencia" de Rectores, estudiantes y parlamentarios de la Comisión de Presupuesto de la Cámara de Diputados, con el fin de acercar posiciones en el marco de la discusión presupuestaria 2013. ${ }^{27}$

26 Esta convergencia ocurrió en 17 de los 18 puntos planteados por los Rectores. El único punto no suscrito por los Estudiantes fue la finalización de las movilizaciones.

27 Un nuevo tema fue posicionado por el CRUCH, mediante su rechazo del Proyecto de Ley preparado por el Gobierno para 
En la perspectiva estructural, es decir, de observación sobre los componentes de funcionamiento del Sistema de Educación Superior, es donde se concentran los aspectos centrales del conflicto sociopolítico, en torno de la 'transformación' del principio de subsidiariedad, en este aspecto asociado al autofinanciamiento de las universidades. Por esto, los puntos críticos abordados por las propuestas de Reforma de la Educación son:

- Instrumentos de financiamiento y regulación del "Sistema Nacional deEducación" o "Sistema Público de Educación Gratuita para Chile”, aumento de los aportes basales del Estado a las Universidades Estatales y Tradicionales, eliminación del Aporte Fiscal Indirecto y la creación de la Superintendencia de Educación.

- El patrón histórico de relación entre Universidades Estatales y el Estado que, desde la perspectiva de los rectores establece una distinción de lo público-estatal, respecto de Universidades Tradicionales y señala Compromisos de las Universidades (Calidad con equidad, apoyo al Estado, provisión de opciones de Educación Superior para todos, constituirse en modelo de institución pública, crear y mantener espacios públicos); de la sociedad (Crear un aporte basal permanente, 50\% del presupuesto anual de cada Universidad; Consejo Nacional de Educación Estatal para evaluación de Resultados; legislar para dotar a las universidades de un marco legal adecuado ${ }^{28}$ y entre las Universidades y el Estado (cuentas públicas transparentes y responsabilidad en el uso de recursos públicos, mediante el logro de desempeños de excelencia).

- La Educación Pública como instrumento de un Estado Garantista del Derecho a la Educación.

reformar la institucionalidad de investigación con el traspaso de la Comisión Nacional de Investigación Científica y Tecnológica (CONICYT) al Ministerio de Economía, con el fin de estrechar el vínculo entre ciencia y aparato productivo nacional. Al respecto, los Rectores señalaron "El Consejo de Rectores manifiesta que Conicyt, Consejo de Ciencia y Tecnología, debe ser independiente de los vaivenes políticos y mantener su estrecha relación con todas las instituciones que desarrollan investigación en Ciencia y Tecnología y forman el capital humano avanzado que el país requiere [...] rechazamos la medida de trasladar Conicyt al Ministerio de Economía porque daña el desarrollo de las ciencias, la formación de investigadores y el vínculo con el Sistema de Educación Superior". Consejo de Rectores de las Universidades Chilenas. Declaración Pública del CRUCH (Santiago: CRUCH, 2012). Consulta 5 de noviembre de 2012 http://www.consejoderectores.cl/site/detalle-noticias-declaracion_publica-147.html

28 Esto implicaría modificar la Ley General de Educación del año 2009 y el conjunto de Decretos con Fuerza de Ley promulgados entre 1980-1990, que determinaron el autofinanciamiento de las Universidades y la privatización de la Educación Superior. 
Desde la perspectiva de proceso, lo que se aprecia es la crítica y reforma de las transformaciones neoliberales de la Educación, ocurridas tras el golpe militar de 1973, que definieron la modernización mediante la implementación de lo que J. J. Brunner y Daniel Uribe denominan el mercado universitario. En este punto, lo que se aprecia son los elementos irreversibles en el campo educacional chileno; por eso, su modificación implica ir de la reforma educativa-universitaria a la reforma social. Los tópicos aquí considerados son:

- Lograr que prime el Derecho a la Educación por sobre la Libertad de Enseñanza, mediante reformas a la Constitución de 1981, lo que, en la perspectiva del movimiento estudiantil, implica una reorganización del Sistema de Educación Superior, o de complementariedad entre las propuestas del Nuevo Trato entre Universidades y el Estado, mediante la Superintendencia de Educación Superior que se propuso crear.

- La idea de Excelencia en la enseñanza universitaria, vinculada tanto a los requerimientos productivos del país como a las denominadas "necesidades sociales", lo que implicaría un cambio cultural referido al sentido de la educación y las lógicas de profesionalización que le son consustanciales.

- Autonomía universitaria, como una delimitación del espacio públicoestatal, diferenciado de los particularismos e intereses privados. En este sentido, la autonomía no solo está definida como la protección respecto de los intereses de lucro, los requerimientos del "mundo empresarial-productivo" -o de los "hombres de negocios", como señaló T. Veblen- sino que también requiere de un rol activo del Estado mediante el respeto por la condición de la Universidad como actor del desarrollo nacional y de la formación de seres humanos respetuosos de los valores societales que aseguran la cohesión e integración. ${ }^{29}$

29 Así se planteó: "Para garantizar la autonomía, la educación pública debe contar con una organización interna capaz de auto determinarse en las esferas administrativas, de gobierno, técnico-docente y financiera". Comisión de Difusión y Cultura Escuela de Trabajo Social Universidad de Valparaíso. Principios para una reforma de la Educación Superior, (Valparaíso: Universidad de Valparaíso, 2011). Consulta 5 de noviembre de 2012 http://comisiondifusiontsuv.blogspot.com/2011/05/principios-para-unareforma-la.html 


\section{CONCLUSIÓN}

En estas notas se ha propuesto un conjunto de antecedentes que, articulados en el mismo contexto de conflictividad sociopolítica entre el movimiento social-universitario y el Estado, configuran una perspectiva que reconoce en el 'problema educativo' planteado un ámbito de politización, crítica y reforma del sistema educación superior y de la sociedad. La coyuntura de movilizacón trascendió los límites de funcionamiento del subsistema educativo, la gobernabilidad neoliberal basada en la separación entre economía, política y cultura fue puesta entre paréntesis por el movimiento social-universitario.

Es necesario puntualizar la importancia que tuvo la Univerisdad de Chile en la lógica de reforma desplegada en el escenario descrito. El planteamiento de una Educación Pública tuvo soporte histórico, político y discursivo en el fortalecimiento y protagonismo de la Universidad que reivindica su tradición, su carácter nacional y público; todo lo que configura su singular vínculo con el Estado. La reivindicación del rol de la Universidad se basó en su capacidad institucional, contextual, histórica, de 'comprender' las necesidades de desarrollo del país y de disponer, en una lógica de globalización, de los recursos cognitivos para responder a tales requerimientos. Este fue un discurso que ofreció cohesión, propuso diagnósticos, interpeló al Estado y, sobre todo, instó a la unidad de actores que hicieron un cambio en la política del movimiento social-universitario: Rectores y Estudiantes.

Lo anterior determinó en gran medida el carácter del cambio político que impulsó el movimiento social-universitario. La generación de liderazgos fue inscrita en un contexto de movilización y en la ampliación progresiva del conflicto desde la 'problemátiva educativa' a la crítica de los fundamentos culturales de la sociedad chilena y su lógica neoliberal hegemónica. No obstante, es necesario recalcar que esta 'politización' siguió derroteros novedosos que se sumaron a las propias transiciones culturales y generacionales de la expresividad de la protesta. Tales mediaciones se instalaron en la interpelación al Estado y su abandono de la garantización del derecho a la educación, argumentando la hegemonía de la Libertad de Enseñanza, la denuncia del lucro en el sistema educativo en general y, por ello, la necesidad de cambios estructurales que solo podían tener rango constitucional. 
De consuno, el conflicto se hizo político porque instaló un espacio público propio -la calle, las universidades, los colegios, las redes sociales, los medios de comunicación- que se nutrió de intervenciones orientadas en una línea de integración común: la Educación como bien público.

Así, la problematización de la noción y/o principio de autonomía universitaria como ámbito de reivindicación, fortaleciemiento y desarrollo de Universidad Estatal-Pública, en el contexto actual del Sistema de Educación Superior en Chile, se inscribió en una política compleja. Antes que una reformulación del mentado pacto histórico, la Universidad se resposicionó como un actor sistémico distinguido por su carácter público y, por ello, que debería ser considerado en la formulación de los criterios fundamentales de la política educacional, provisto de la posición y recursos particulares en el campo de aplicación de una política social, y evaluado diferencialmente respecto de instituciones universitarias privadas. Esto lleva a pensar en cuánto y cómo favorece el discurso de la calidad educativa a la reforma social, que concretaría el cambio político que el movimiento social-universitario le ha propuesto a la sociedad chilena.

\section{FUENTES}

Consejo de Rectores de las Universidades Chilenas. Declaración Pública del CRUCH. Santiago: CRUCH, 2012.

Confederación de Estudiantes de Chile. Historia de la Confech y el movimiento universitario chileno en los últimos 30 años. Santiago: Confech, 2011.

Confederación de Estudiantes de Chile. Fundamentos para un sistema público gratuito de educación para Chile. Santiago: Confech, 2011.

\section{REFERENCIAS}

Acevedo, Álvaro. “Una heurística para el estudio de la historia de las reformas y conflictos en la universidad latinoamericana". Revista Historia de la Educación Colombiana 5 (2005): 177-188.

Brunner, José Joaquín. Educación Superior en Chile. Instituciones, mercados y políticas gubernamentales (1967-2007), Santiago: Ediciones Universidad Diego Portales, 2009. 
Brunner, José Joaquín y Uribe, Daniel. Mercados universitarios: el nuevo escenario de la educación superior. Santiago: Ediciones Universidad Diego Portales, 2007.

Confederación de Estudiantes de Chile. Fundamentos para un sistema público gratuito de educación para Chile. Santiago: Confech, 2011. Consulta 5 de noviembre de 2012 http://www. fepucv.cl/wp-content/uploads/2012/04/FUNDAMENTOS-POLITICOS-DEFINITIVO.pdf

Consorcio de Universidades del Estado de Chile. Nuevo trato con el Estado. Fortaleciendo la política de Educación Superior Estatal. Santiago: Consorcio de Universidades del Estado de Chile, 2009. Consulta 5 de noviembre de 2012 http://www.uestatales.cl/cue/?q=node/2234

Donati, Pierpaolo. "Nuevas políticas sociales y Estado social relacional". Revista Española de Investigaciones Sociológicas 108 (2004): 9-47.

Espinoza, Oscar y González, Luis. "Políticas de Educación Superior en Chile desde la perspectiva de la Equidad". Sociedad y Economía 22 (2012): 69-94.

Fairclough, Norman. "El análisis crítico del discurso y la mercantilización del discurso público: las universidades", Revista Discurso y Sociedad, Vol. 2, 1 (2008). Consulta 30 de octubre de 2012 http://www.dissoc.org/ediciones/v02n01/DS2\%281\%29Fairclough.pdf

Insunza, Jorge. La construcción del derecho a la Educación y la institucionalidad educativa en Chile. Santiago de Chile: OPECH, 2009.

Lazzarato, Mauricio. Le gouvernement des inégalités. Critique de l'insécurité néolibérale. París: Éditions Amsterdam, 2008.

Lechner, Norbert. “El debate sobre Estado y Mercado”, Nueva Sociedad 121 (1991).

Lyotard, J.F. La condición posmoderna. Informe sobre el saber. Madrid: Editorial Cátedra, 1987.

Mayol, Alberto. El derrumbe del modelo. La crisis de la economía de mercado en el Chile contemporáneo. Santiago: LOM, 2012.

Molina, José. “La mediación social de la Universidad”. Revista América Latina12 (2011): 179208.

Restrepo, Manuel. "Ratificar que la Universidad es real, librepensadora, autónoma... es nuestra". Memorias Primer Encuentro Grupos de Investigación en la Facultad de Ciencias de la Educación. Tunja: CIEFED-UPTC, 2012.

Ruiz, Carlos. De la República al mercado. Ideas educacionales y política en Chile. Santiago: LOM, 2010.

Sader, Emir, Pablo Gentili y Hugo Aboites. La Reforma Universitaria: desafíos y perspectivas noventa años después. Buenos Aires: CLACSO, 2008.

Seoane, José, Clara Algranati y Emilio Taddei. "Realidades y desafíos políticos de Nuestra América". Revista América Latina 11, (2011). 
Serrano, Sol. Universidad y Nación. Chile en el siglo XIX. Santiago: Editorial Universitaria, 1994.

Tilly, Charles. "Conflicto político y cambio social". En Los movimientos sociales. Transformaciones políticas y cambio cultural, editado por Pedro Ibarra y Benjamín Tejerina 25 - 42. Madrid: Editorial Trotta, 1998.

\begin{tabular}{|l|} 
\\
\hline Molina Bravo, José. “Educación pública, autonomía universitaria y cam- \\
bio político: Notas para el análisis del movimiento universitario en Chile, \\
2011”. Revista Historia de la Educación Latinoamericana. Vol. 15 No, 21, \\
(2013): 263 - 282.
\end{tabular}




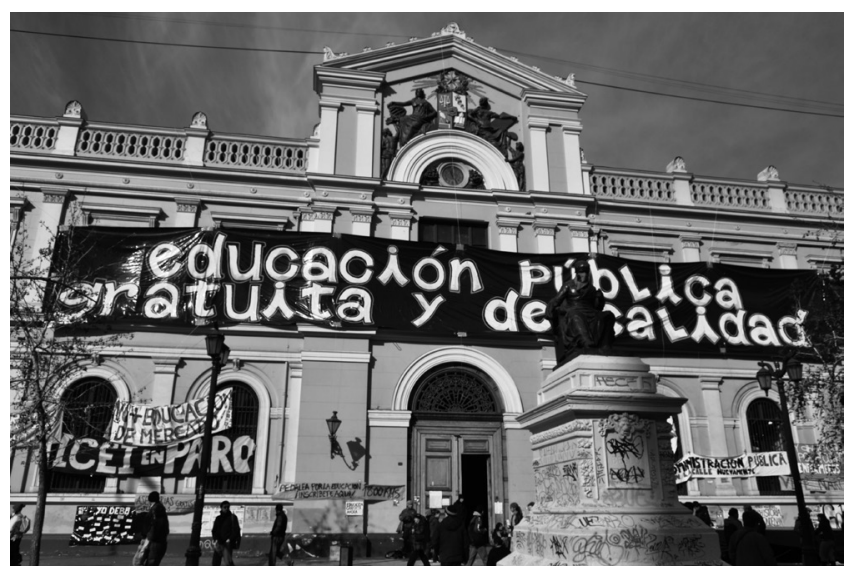

Fuente: Casa - Central de la Universidad de Chile - frontisSantiago.

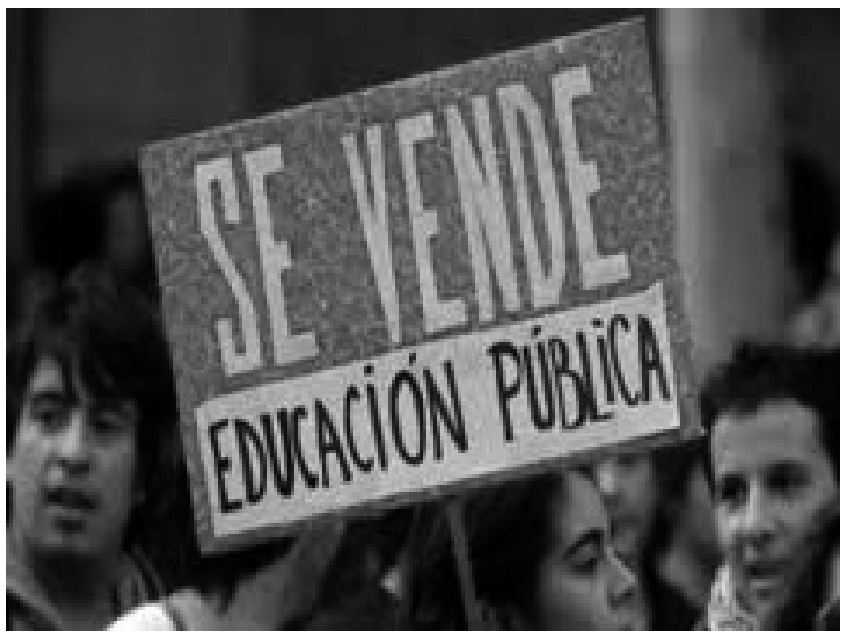

Fuente: Protestas estudiantes de Chile 2011. 